\title{
Comunicación
}

\section{Infestación por Toxocara cati en un ejemplar de Leopardus pardalis en Colombia: reporte de caso}

\author{
Toxocara cati infestation in a Leopardus pardalis specimen in \\ Colombia; case report
}

\author{
Fabián Andrés Muñoz-Rodriguez ${ }^{1,2}$, Samir Ramírez Gutiérrez ${ }^{2}$, \\ Lilibeth Doria Pérez ${ }^{3}$, Daniel Leonardo Cala-Delgado ${ }^{4.5}$
}

\section{Resumen}

Se presenta el caso de un espécimen de Leopardus pardalis, macho y peso de 15.6 $\mathrm{kg}$, hallado con descomposición parcial en una vía del sector rural del municipio de Arauca, Colombia. En la necropsia se encontraron 36 formas parasitarias de nematodos, con una longitud aproximada de $3.4 \mathrm{~cm}$, las cuales fueron identificadas como Toxocara cati por la presencia de aletas laterales en el extremo cervical, presencia de tres labios y de una cutícula anillada. Este es el primer reporte en Colombia de la especie parasitaria en este felino silvestre, indicando que la especie L. pardalis puede ser potencial portador y diseminador de $T$. cati.

Palabras clave: felino silvestre, nematodo, Orinoquia, parásitos

\section{AbSTRaCT}

It is reported the case of a Leopardus pardalis specimen, male, $15.6 \mathrm{~kg}$ body weight, found with partial decomposition in a road in the rural sector of the municipality of Arauca, Colombia. At necropsy, 36 parasitic forms of nematodes were found, with an

${ }^{1}$ Grupo de Investigaciones Los Araucos, Facultad de Medicina Veterinaria y Zootecnia, Arauca, Colombia

${ }^{2}$ Semillero de Investigación Cabalgando, Universidad Cooperativa de Colombia, sede Arauca, Arauca, Colombia

${ }^{3}$ Instituto Colombiano Agropecuario - ICA, Arauca, Colombia

${ }^{4}$ Grupo de Investigación en Ciencias Animales, Universidad Cooperativa de Colombia (GRICA - UCC) Bucaramanga, Colombia

${ }^{5}$ E-mail: daniel.cala@campusucc.edu.co

Recibido: 16 de junio de 2020

Aceptado para publicación: 15 de enero de 2021

Publicado: 24 de abril de 2021 
approximate length of $3.4 \mathrm{~cm}$, which were identified as Toxocara cati by the presence of lateral fins at the cervical end, the presence of three lips and a ringed cuticle. This is the first report in Colombia of the parasitic species in this wild feline, indicating that the species $L$. pardalis may be a potential carrier and disseminator of $T$. cati.

Key words: wild feline, nematode, Orinoquia, parasites

\section{INTRODUCCIÓN}

América del Sur contiene una amplia diversidad de carnívoros terrestres, reportándose más de 29 especies (Prevosti et al., 2007). En Colombia se encuentran 6 de las 36 especies de felinos silvestres reportadas a nivel mundial (Panthera onca, Puma concolor, Leopardus pardalis, leopardus wiedii, Puma yaguarundi y Leopardus tigrinus), distribuidos desde las selvas amazónicas hasta los ecosistemas de la región andina y Orinoquia colombiana (PayánGarrido y Soto-Vargas, 2017).

Tigrillo o cunaguaro son nombres comunes del Leopardus pardalis dentro del territorio colombiano. Este es un felino neotropical de tamaño mediano (Nowell y Jackson, 1996), que habita por debajo de los $1200 \mathrm{msnm}$ (Dillon y Kelly, 2007), aunque se dispone de registros a más de $3000 \mathrm{msnm}$ (Jiménez et al., 2010). Se alimenta principalmente de pequeños roedores y reptiles. Si bien su estado de conservación no es alarmante, algunas poblaciones están en peligro de extinción (Janeèka et al., 2011).

Parásitos apicomplejos intracelulares como Hepatozoon spp, Toxoplasma gondii y Sarcocystis spp afectan a los felinos, incluyendo las especies de vida silvestre (Kenny et al., 2002; Cañón et al., 2016; De Sousa et al., 2017). Asimismo, los parásitos gastrointestinales llegan a causar disminución del apetito, anemia y pérdida de proteínas plasmáticas en el tracto gastrointestinal, entre otros (Aquino et al 2009).
Los gatos son los huéspedes definitivos de $T$. cati, cuyo ciclo de vida es similar al de T. canis, aunque no se transmite por vía intrauterina y los cachorros solo se infectan por la leche o el calostro si la gata se infecta de manera aguda en la última etapa de la gestación (Archelli y Kozubsky, 2008). T. cati es considerada como una zoonosis parasitaria de distribución mundial (Martýìnez et al., 2003), por la convivencia entre humanos y animales domésticos (Despommier, 2003). La infestación por migración larvaria de Toxocara sp se le asocia con dos síndromes clínicos en el humano, la larva migrans ocular (LMO) y la larva migrans viceral (LMV) (Pawlowski, 2001). La defecación incontrolada de animales infectados produce contaminación del suelo y áreas verdes por huevos de Toxocara (Martínez et al., 2003). Son pocos los hallazgos donde este parásito infestan a felinos silvestres. Por tal motivo, el presente reporte documenta la presencia del nematodo $T$. cati en felinos silvestres en un individuo de la especie Leopardus pardalis en Colombia.

\section{REPORTE}

El presente trabajo se realizó con un ejemplar L. pardalis hallado por campesinos el 17 de mayo de 2017 en una de las vías del sector rural del municipio de Arauca, en Arauca, Colombia. El cadáver fue llevado a los laboratorios de morfofisiología de la granja El Picure, Facultad de Medicina Veterinaria y Zootecnia de la Universidad Cooperativa de Colombia, campus Arauca. Los análi- 
Cuadro 1. Peso de los órganos internos del Leopardus pardalis y su relación en (\%) con el peso total del mismo.

\begin{tabular}{lcc}
\hline Órgano & $\begin{array}{c}\text { Peso } \\
(\mathrm{kg})\end{array}$ & $\begin{array}{c}\text { Índices viscerosomáticos } \\
(\%)\end{array}$ \\
\hline Corazón & 0.265 & 1.7 \\
Pulmones & 1.46 & 9.4 \\
Tráquea & 0.22 & 1.4 \\
Esófago & 0.26 & 1.7 \\
Estómago & 0.84 & 5.4 \\
Intestino delgado & 0.79 & 5.1 \\
Intestino grueso & 0.30 & 2.0 \\
Hígado & 1.34 & 8.6 \\
Páncreas & 0.28 & 1.8 \\
Cerebro & 0.38 & 2.5 \\
Riñones & 0.25 & 1.6 \\
Vejiga & 0.10 & 0.7 \\
Bazo & 0.18 & 1.2 \\
\hline
\end{tabular}

sis post mortem se realizaron siguiendo las técnicas descritas por Caicedo et al. (2012) y Peleteiro (2016).

El cadáver debía tener alrededor de 24 horas del deceso debido a las características del rigor mortis. Tenía un peso de $15.6 \mathrm{~kg}$, y se encontraba sin piel, orejas, nariz y garras, y con signos primarios de putrefacción principalmente en la región abdominal. El cadáver presentaba un corte en la región atlantooccipital, lo que presuntamente le causó la muerte por decapitación con arma cortopunzante; a su vez presentaba una contusión en la región temporo-parietal izquierda. Los órganos fueron pesados y se determinaron los índices viscerosomáticos (Cuadro 1). Para esto, se pesó todo el animal y posteriormente se pesó cada uno de los órganos descritos (Schuingues et al., 2019).
Se registraron las medidas morfométricas y peso de los órganos y se tomaron muestras de mucus y restos de alimento del tracto gastrointestinal. Las formas parasitarias adultas fueron conservadas en alcohol al $65 \%$. Para el reconocimiento y caracterización de las formas parasitarias se colocaron en cajas de Petri con alcohol al $65 \%$ y se observaron al estereoscopio y microscopio a 10x, 30x y 40x tomando las técnicas descritas por Serrano (2010) y Pérez e Iglesias (2008). Las muestras de alimento y mucus fueron analizadas en el microscopio a $10 \mathrm{X}$ y $40 \mathrm{X}$, con el fin de descartar estadios larvarios de diferentes parásitos.

En el duodeno y yeyuno se encontraron 36 formas parasitarias (Figura 1), identificadas como Toxocara cati por la presencia de aletas cervicales (Figura 2), esófago con bul- 


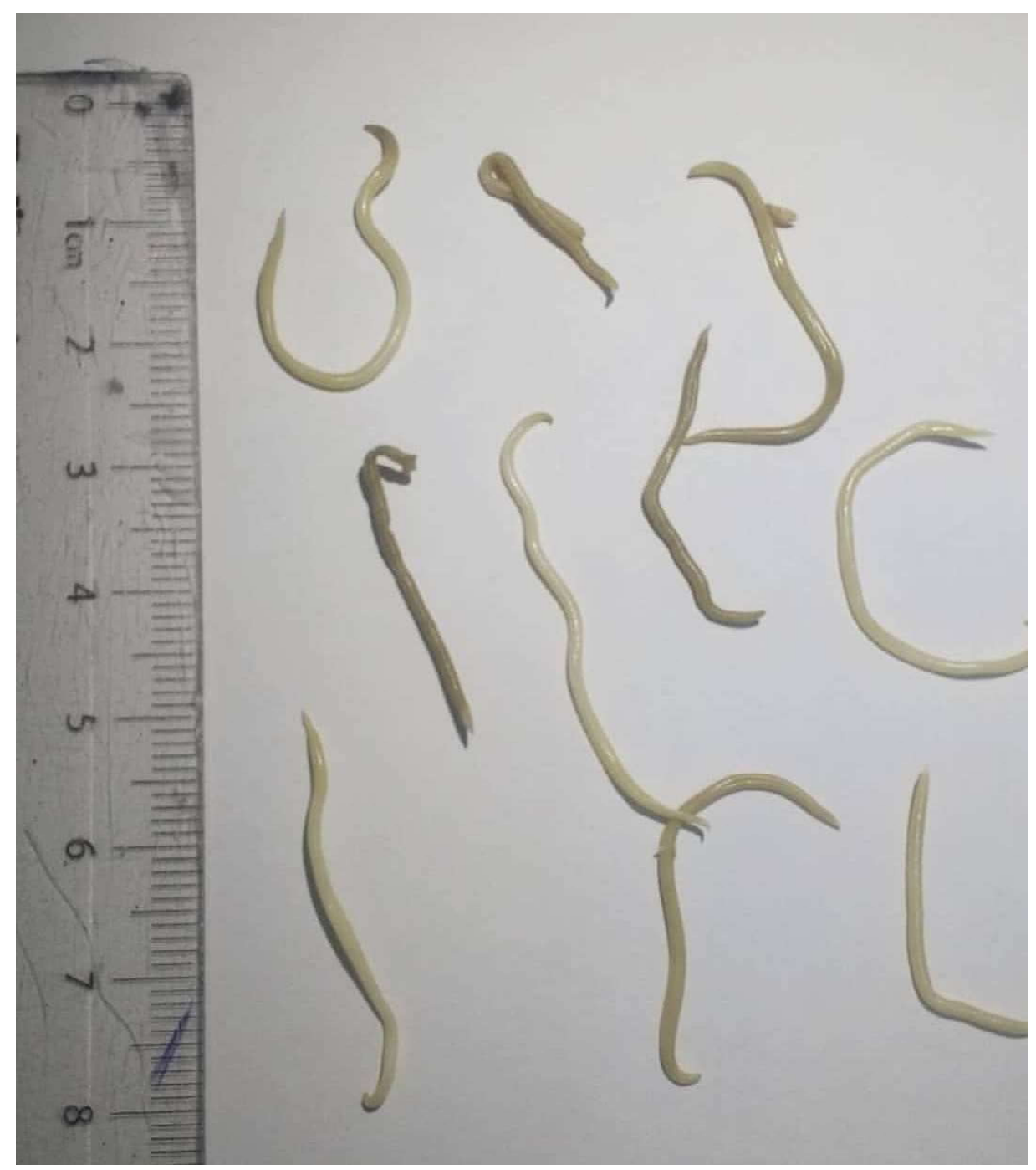

Figura 1. Observación macroscópica de parásitos intestinales encontrados en un espécimen de Leopardus pardalis (Arauca, Colombia)

bo en el extremo posterior, machos con un apéndice terminal y hembras con una vulva en el tercio anterior del cuerpo (Delgado y Rodríguez, 2009). Presentaban entre 1.5 y $6.7 \mathrm{~cm}$ de largo, con un promedio de $3.4 \mathrm{~cm}$. Estas medidas de T. cati fueron similares a las reportadas por Pérez e Iglesias (2008) y Gallas y da Silveira (2011).

\section{Discusión}

T. cati es uno de los helmintos más frecuentes en gatos. Coati (2002) demostró que gatos con circulación libre, sobre todo en áreas rurales, se encuentran más frecuentemente infestados por ascaridos que sus congéneres 


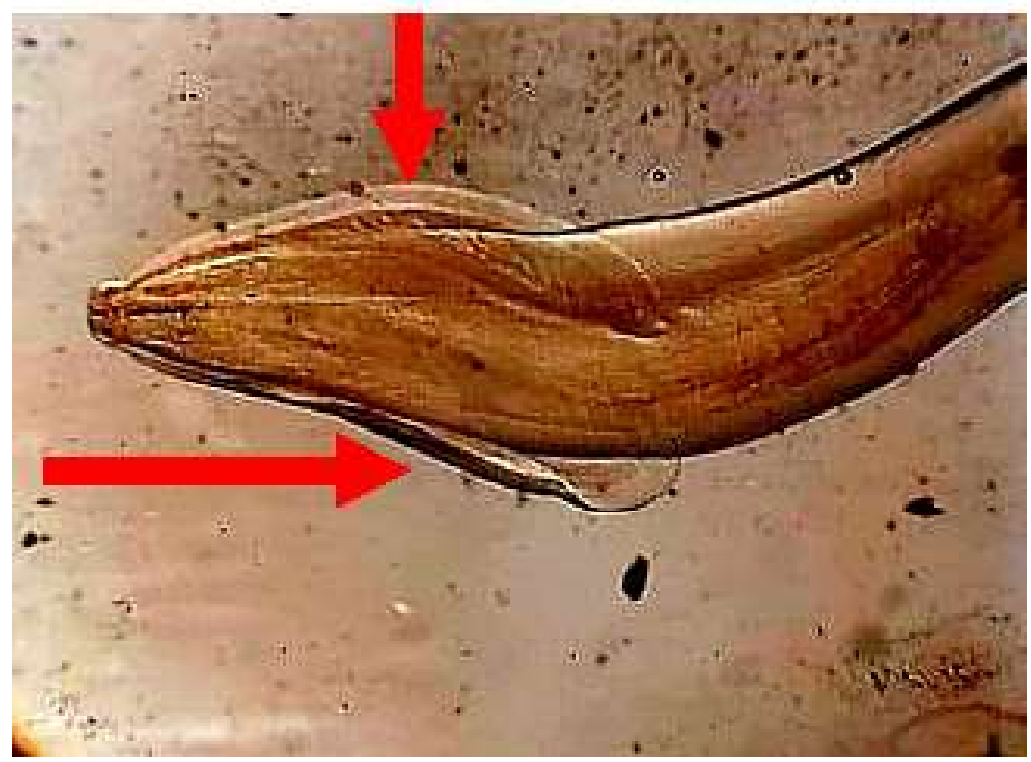

Figura 2. Región anterior de Toxocara cati adulto, con extensiones alargadas de la cutícula, asas cervicales (flechas) encontrados en un espécimen de Leopardus pardalis (Arauca, Colombia)

de la ciudad y gatos residentes en hogares familiares. Una de las vías de infección es a través de hospedadores paraténicos como los roedores o la infestación galactógena de $T$. cati: Este nematodo es determinante desde el punto de vista epidemiológico, ya que puede persistir por más de seis meses en su hospedador (Swerczek et al., 1971; Bowman, 2008). Gallas y da Silveira (2013) encontraron formas parasitarias de $T$. cati en 17 felinos silvestres (Leopardus colocolo, Leopardus geoffroyi, Lentinus tigrinus, Puma yagouaroundi) que murieron atropellados. Varios de los reportes son de animales de zoológicos, los cuales están en contacto directo con otros vectores que facilitan la transmisión de este parásito, en tanto que el presente reporte es el primero de un animal silvestre parasitado con $T$. cati en Colombia.
En vista que el hospedero definitivo de este nematodo es el gato doméstico y teniendo en cuenta las similitudes en sus sistemas digestivos, sumado a la alta carga parasitaria que presentaba (36 nematodos adultos), puede inferirse que Leopardus pardalis es un potencial hospedero de T. cati. Una hipótesis que explica estos acontecimientos de infestación, son los efectos generados por amenazas transversales que viven todas las especies de felinos silvestres, como la pérdida y transformación del hábitat causada por el avance de la frontera agropecuaria, la fragmentación de sus poblaciones por carreteras y poblados etc, (Sarmiento et al., 2016) y con esto la falta de presas silvestres para cazar, lo que genera que estos felinos se vean obligados a acercarse a los asentamientos humanos con fines alimenticios. 
La gran problemática existente entre la relación hombre-felino se han visto tradicionalmente como un tema de depredación ocasionado por los felinos silvestres cuando atacan los animales de producción, como ocurre en las sabanas del departamento de Arauca. Estos casos se relacionan en gran medida con predios cercanos a áreas protegidas, en el límite de zonas boscosas, fincas con alta densidad de animales de producción extensiva, como porcino u ovinos y áreas con poco número de presas naturales (Corrales-Gutiérrez et al., 2016).

\section{Literatura Citada}

1. Aquino LH, Yamada FH, Ceschini TL, Takemoto RM, y de Carvalho Pinto CJ 2009. Study of the gastrointestinal parasites in felines from Itaipu Binacional wild animal nursery, Brazil. Arch Cienc Vet Zool UNIPAR 12: 11-15.

2. Archelli S, Kozubsky L. 2008. Toxocara y toxocariosis. Acta Bioquím Clín Latinoam 42: 379-384.

3. Bowman DD, Hendrix CM, Lindsay DS Barr SC 2008. Parasitología clínica felina. USA: John Wiley. 469 p.

4. Caicedo J, Ospina J. C, Avila J. 2012. Técnica de necropsia, interpretación de hallazgos macroscópicos y toma de muestras en mamíferos silvestres. En: Conferencia Interna en Medicina y Aprovechamiento de Fauna Silvestre, Exótica y no Convencional. Cuba.

5. Cañón-Franco WA, López-Orozco N, Christoff $A U$, de Castilho CS, de Araújo FA, Verma SK, Dubey JP, et al. 2016. Molecular and morphologic characterization of Sarcocystis felis (Apicomplexa: Sarcocystidae) in South American wild felids from Brazil. Vet Parasitol 217: 15-20. doi: 10.1016/ j.vetpar.2015.12.025
6. Coati N. 2002. Pränatale und galaktogene Infektionen mit Toxocara cati SCHRANK 1788 (Anisakidae) bei der Katze. Vet. Med. Diss. Germany: Hannover University. $77 \mathrm{p}$.

7. Corrales-Gutiérrez D, Salom-Pérez R, Hoogesteijn R. 2016. Implementación de estrategias anti-depredadoras en ranchos ganaderos ubicados dentro de los corredores biológicos importantes de Costa Rica. En: Conflicto entre felinos y humanos en América Latina. Bogotá, Colombia: Instituto de Investigación de Recursos Biológicos Alexander von Humboldt. p 152-167.

8. de Sousa KC, Fernandes MP, Herrera HM, Benevenute JL, Santos FM, Rocha FL, Barreto WT, et al. 2017. Molecular detection of Hepatozoon spp in domestic dogs and wild mammals in southern Pantanal, Brazil with implications in the transmission route. Vet Parasitol 237: 37-46. doi: 10.1016/j.vetpar.2017.02.023

9. Delgado O, Rodríguez-Morales AJ. 2009. Aspectos clínicos y epidemiológicos de la toxocariasis: una atención insatisfecha en Venezuela y América Latina. Bol Mal Salud Amb 49: 1-33.

10. Despommier D. 2003. T oxocariasis: clinical aspects, epidemiology, medical ecology and molecular aspects. Clim Microb Rev 16: 265-272.

11. Dillon A, Kelly MJ. 2007. Ocelot Leopardus pardalis in Belize: the impact of trap spacing and distance moved on density estimates. Oryx 41: 469-477. doi: 10.1017/S0030605307000518

12. Gallas M. da Silveira EF 2011. Análise da variação morfológica entre caracteres de Toxocara cati (Nematoda, Ascarididae) coletados de felídeos silvestres no Rio Grande do Sul, Brasil.Rev Iniciación Cient ULBRA 9: 31-39.

13. Gallas M, da Silveira EF. 2013. Toxocara cati (Nematoda, Ascarididae) in different wild feline species in Brazil: new host records. Biotemas 26: 117-125. 
14. Janeèka JE, Tewes ME, Laack LL, Caso A, Grassman LI, Haines AM, Shindle DB, et al. 2011. Reduced genetic diversity and isolation of remnant ocelot populations occupying a severely fragmented landscape in southern Texas. Anim Conserv 14: 608-619. doi: 10.1111/ j.1469-1795.2011.00475.x

15. Jiménez CF, Quintana H, Pacheco V, Melton D, Torrealva J, Tello G. 2010. Camera trap survey of medium and large mammals in a montane rainforest of northern Peru. Rev Peru Biol 17: 191-196.

16. Kenny DE, Lappin MR, Knightly F, Baler J, Brewer M, Getzy DM. 2002. Toxoplasmosis in Pallas' cats (Otocolobus felis manul) at the Denver Zoological Gardens. J Zoo Wildlife Med 33: 131-138. doi: 10.1638/1042-7260(2002)033[0131:TIPCOF]2.0.CO;2

17. Martýinez-Barbabosa I, Tsuji OV, Cabello RR, Cárdenas EMG, Chasin OA. 2003. The prevalence of Toxocara cati in domestic cats in Mexico City. Vet Parasitol 114: 43-49. doi: 10.1016/S03044017(03)00038-4

18. Nowell K, Jackson P. 1996. Wild cats: status survey and conservation action plan. Gland, Switzerland: IUCN. 382 p.

19. Pawlowski Z. 2001. Toxocariasis in humans: clinical expression and treatment dilemma. J Helminthol 75: 299305. doi: $10.1017 / \mathrm{s} 0022149 \times 01000464$

20. Payán-Garrido E, Soto-Vargas $C$. 2012. Los felinos de Colombia. Bogotá, Colombia: Ministerio de Ambiente y Desarrollo Sostenible. $48 \mathrm{p}$.
21. Peleteiro M, Silva J, Dias-Pereira P, Carvalho T, Faustino A, Pissara $H$. 2016. Manual de necrópsia veterinária. Lisboa, Portugal: LIDEL. $180 \mathrm{p}$.

22. Pérez-Tort G, Iglesias MF, Más J. 2008. Atlas de parasitología en pequeños animales. Inter-Médica. $80 \mathrm{p}$.

23. Prevosti JF, Soibelzon LH, Pons G, Vicens D, Cuerda J. 2007. Los carnívoros (Carnivora, mammalia) terrestres del Cuaternario de América del Sur. En: Pons GX, Vicens D (eds). Geomorfologia Litoral i Quaternari. Homenatge a Joan Cuerda Barceló. España: Societat d'Història Natural de Les Balears. p 51-68.

24. Sarmiento-Giraldo MV, Sánchez-Palomino P, Monroy-Vilchis O. 2016.Depredación de ganado por jaguar (Panthera onca) y puma (Puma concolor) en las sabanas inundables de Arauca y Casanare, Colombia. En: Conflictos entre felinos y humanos en América Latina [Conflictos entre felinos y humanos en América Latina. Bogotá, Colombia: Instituto de Investigación de Recursos Biológicos Alexander von Humboldt. p 103-121.

25. Serrano-Aguilera FJ. 2010. Manual práctico de parasitología veterinaria. España: Univ. de Extremadura. 115 p.

26. Schuingues CO, Lima MG, SantosFilho M, Campos DVS, Costa GM. 2019. Características morfológicas do estômago de jaguatirica (Leopardus pardalis - Linnaeus, 1758). Arq Bras Med Vet Zootec 71: 1171-1178 doi: 10.1590/1678-4162-10584

27. Swerczek TW, Nielsen SW Helmboldt CF. 1971. Transmammary passage of Toxocara cati in the cat. Am J Vet Res 32: 89-92. 